\title{
Antibiotic Susceptibility Patterns of Salmonella Typhi and Salmonella Paratyphi in a Tertiary Care Hospital in Islamabad
}

\author{
Masab Umair ${ }^{1}$, Shajee Ahmad Siddiqui ${ }^{1}$ \\ 1. General (Internal) Medicine, Pakistan Institute of Medical Sciences, Shaheed Zulfiqar Ali Bhutto Medical University, \\ Islamabad, PAK
}

Corresponding author: Masab Umair, masab.umair.rmc.36@gmail.com

\section{Abstract \\ Background}

Enteric fever is a serious public health problem in Pakistan. Growing problem of drug-resistant Salmonella strains and outbreak of ceftriaxone-resistant Salmonella typhi in Hyderabad during 2016-2017 is concerning. This study aimed to determine the antibiogram profile of Salmonella typhi and Salmonella paratyphi isolated from blood cultures of patients presenting in Pakistan Institute of Medical Sciences (PIMS), Islamabad.

\section{Materials and methods}

A retrospective cross-sectional study conducted in PIMS. A case of enteric fever was defined as a patient with blood culture positive for either S. typhi or S. paratyphi. Demographics and antibiogram profile of the 664 cases who presented during 2012-2018 were included in this study.

\section{Results}

Out of 664 cases, S. typhi was isolated from 528 and S. paratyphi was isolated from 136 cases. Males accounted for the majority of the cases $(n=440,66.3 \%)$. Clustering of the cases was observed in young adults (18-25 years). Incidence was highest during months of summer and monsoon (May-September). Most of the S. typhi isolates were resistant to the first-line antibiotics (amoxicillin 57.6\%, co-trimoxazole 61.4\%, chloramphenicol $46.9 \%$ ) and ciprofloxacin (62.7\%). Antibiotic resistance rates were lowest for imipenem (3.8\%) and ceftriaxone (4.4\%). Among S. typhi isolates tested for all first-line antibiotics, 44.6\% (149/334) were multidrug-resistant (MDR). In contrast, only 12.2\% (11/90) of the S. paratyphi isolates were MDR. 0.7\% $(2 / 283)$ of the tested S. typhi isolates were extensively drug-resistant (XDR). XDR strains were sensitive to imipenem. There was an overall reduction in first-line antibiotic resistance rates from 2012 to 2018.

Received 08/21/2020

Review began $08 / 26 / 2020$ Review ended 08/30/2020 Published 09/03/2020

๑) Copyright 2020

Umair et al. This is an open access article distributed under the terms of the Creative Commons Attribution License CC-BY 4.0., which permits unrestricted use, distribution, and reproduction in any medium, provided the original author and source are credited.

\section{Conclusion}

S. typhi accounted for the majority of the cases of enteric fever. Most S. typhi isolates were resistant to firstline antibiotics. S. paratyphi exhibited lower antibiotic resistance rates. This study recommends thirdgeneration cephalosporins for empirical therapy and for treatment of MDR cases of enteric fever. Imipenem should be reserved for the treatment of XDR Salmonella cases. A decreasing trend in first-line antibiotic resistance rates over time is promising. Antibiotic stewardship is the need of the hour.

Categories: Internal Medicine, Infectious Disease

Keywords: salmonella, typhoid, paratyphoid, enteric fever, multi drug resistant, mdr, extensively drug resistant, xdr

\section{Introduction}

Enteric fever is a disease of intestinal tract caused by Salmonella typhi (typhoid fever) and Salmonella paratyphi (paratyphoid fever). Patients present with fever, malaise, abdominal pain, and constipation. Annually, 21.6 million cases of typhoid fever with 250,000 deaths and 5.4 million cases of paratyphoid fever occur worldwide [1]. Approximately $80 \%$ of the cases and deaths occur in Asia [1]. Incidence of enteric fever in India is from 102-2219/100,000 population [1]. Enteric fever is a major health problem in Pakistan. Estimated annual incidence of S. typhi and S. paratyphi among children in Karachi is 451/100,000 and $76 / 100,000$, respectively [2]. Untreated typhoid fever carries high morbidity and mortality. Without appropriate treatment, typhoid fever carries estimated mortality rate of $30 \%$ [1].

Intestinal perforation is a dreaded complication of typhoid fever [3]. Other complications of typhoid fever include but are not limited to pneumonia, meningitis, endocarditis, osteomyelitis, and arthritis [3]. Presenting symptoms of typhoid fever, therefore, may relate to various organs often resulting in misdiagnosis [3]. Isolation of the organism from the blood culture is vital for the diagnosis of typhoid fever 
[3].

Ampicillin, chloramphenicol, and co-trimoxazole used to be the first-line antibiotics for the treatment of enteric fever [4]. Since the late 1980s, multidrug-resistant strains of Salmonella (MDR, resistant to all firstline antibiotics) have been reported [5]. For quinolone sensitive strains of MDR typhoid, fluoroquinolone is the drug of choice (WHO guidelines 2003) [1]. However, the widespread use of fluoroquinolones has led to the emergence of nalidixic acid-resistant strains with reduced sensitivity to fluoroquinolones in Nepal [5]. In Kolkata and Karachi, approximately $60 \%$ of typhoid fever isolates were found to be nalidixic acid-resistant [6]. This has led to increased use of third-generation cephalosporins. However, third-generation cephalosporin-resistant Salmonella strains have been reported in Nepal and India [1,5]. There has been an outbreak of extensively drug-resistant (XDR) S. typhi in Pakistan during 2016-2017 [7]. This situation is very alarming; clinicians are running out of treatment options and treatment costs have escalated.

Since the emergence of drug-resistant strains of Salmonella, first-line antibiotics fell out of favor and were not frequently used for this illness lately. Interestingly, there have been recent reports of first-line antibiotic susceptible Salmonella strains in Nepal and India $[5,8]$. This re-emergence of first-line antibiotic sensitive Salmonella strains is promising and highlights the importance of continuous antibiogram surveillance.

The aim of this study is to determine the antibiogram profile of S. typhi and S. paratyphi isolated from blood cultures of patients presenting in Pakistan Institute of Medical Sciences. The rationale is to propose appropriate empirical antibiotics for enteric fever in view of increasing drug resistance and evolving antibiograms.

\section{Materials And Methods}

This is a retrospective cross-sectional study that was conducted in Pakistan Institute of Medical Sciences. A case of enteric fever was defined as a patient with blood culture positive for either S. typhi or S. paratyphi. Non-probability consecutive sampling technique was used. This study included the patients aged 12 years and above with blood cultures positive for either S. typhi or S. paratyphi during 2012-2018. Patient's age, gender, date of admission, Salmonella serotype isolated and antibiotic sensitivity profile tested for amoxicillin, azithromycin, cefixime, ceftriaxone, chloramphenicol, ciprofloxacin, cotrimoxazole, imipenem and nalidixic acid were recorded and analysed. MDR was defined as resistance to amoxicillin, chloramphenicol and co-trimoxazole (i.e., first-line antibiotics). XDR was defined as resistance to ciprofloxacin and ceftriaxone (i.e., fluoroquinolones and third-generation cephalosporins) in addition to first-line antibiotic resistance (amoxicillin, chloramphenicol and co-trimoxazole).

\section{Results}

Out of 664 cases included in the study, S. typhi was isolated from 528 (79.5\%) and S. paratyphi was isolated from $136(20.5 \%)$ cases. 440 (66.3\%) were males and 224 (33.7\%) were females with male to female ratio $\approx$ 2:1. Mean age was 25.5 years (range $12-81$ years). Most of the cases belonged to $18-25$ years age group. Age and gender distribution of the cases of S. typhi and S. paratyphi are shown in Table 1. 


\section{Cureus}

Salmonella typhi

n (\%)

146 (27.7)

Total

528

Distribution by gender

Male

Female

342 (64.8)

186 (35.2)

528

Total

136
26 (19.1)

$89(65.4)$

16 (11.8)

5 (3.7)

136

Salmonella paratyphi

n (\%)

Total

172

395

68

29

98 (72.1)

440

38 (27.9)

224

TABLE 1: Age and gender distribution of cases of Salmonella typhi and Salmonella paratyphi

Clustering of the cases was observed during months of summer and monsoon, that is, May-September (Figure 1).

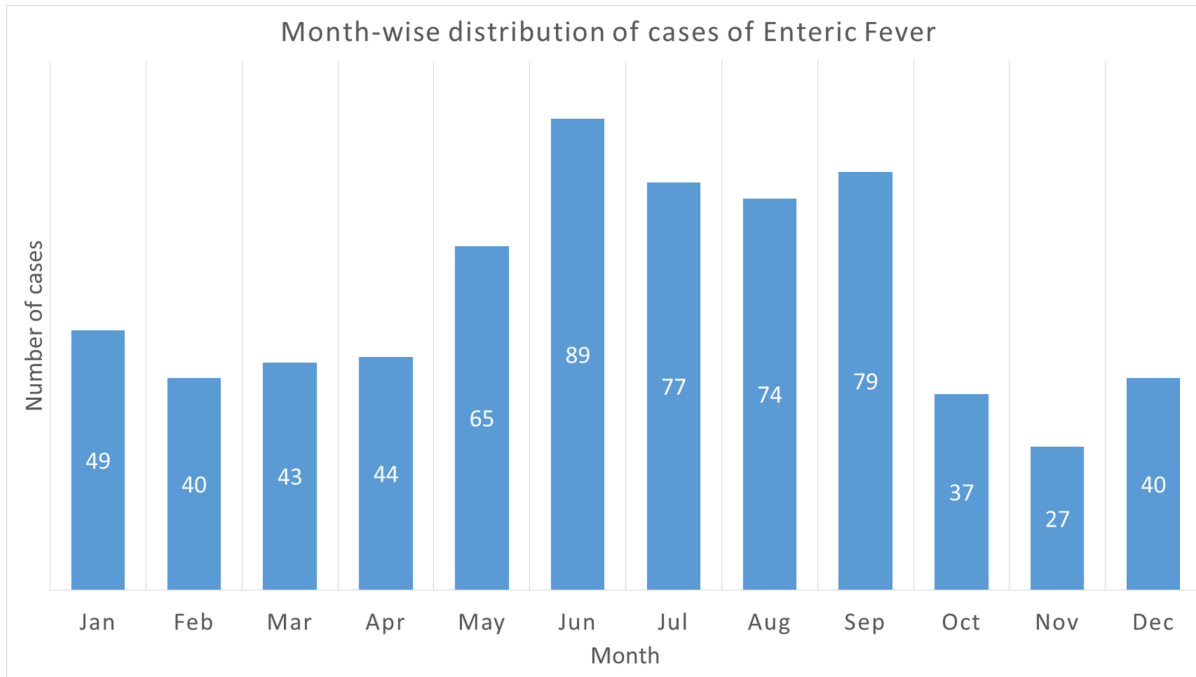

FIGURE 1: Month-wise distribution of cases of enteric fever

Most of the S. typhi isolates were resistant to first-line antibiotics and ciprofloxacin. In general, antibiotic resistance rates were lower in S. paratyphi with exception of azithromycin and ciprofloxacin. Proportion of MDR and XDR strains was higher in S. typhi (Table 2). 


\section{Cureus}

\begin{tabular}{|c|c|c|c|c|}
\hline & \multicolumn{2}{|c|}{ Salmonella typhi } & \multicolumn{2}{|c|}{ Salmonella paratyphi } \\
\hline & Cases tested & Resistant & Cases tested & Resistant \\
\hline & $\mathrm{n}$ & $\mathrm{n}(\%)$ & $\mathrm{n}$ & $n(\%)$ \\
\hline Amoxicillin & 429 & $247(57.6)$ & 1111 & $32(28.8)$ \\
\hline Azithromycin & 322 & 204 (63.4) & 88 & 65 (73.9) \\
\hline Cefixime & 240 & $17(7.1)$ & 42 & $1(2.4)$ \\
\hline Ceftriaxone & 481 & $21(4.4)$ & 123 & $2(1.6)$ \\
\hline Chloramphenicol & 471 & $221(46.9)$ & 125 & $25(20)$ \\
\hline Ciprofloxacin & 475 & $298(62.7)$ & 122 & $86(70.5)$ \\
\hline Co-trimoxazole & 440 & $270(61.4)$ & 117 & $42(35.9)$ \\
\hline Imipenem & 373 & $14(3.8)$ & 103 & $3(2.9)$ \\
\hline Nalidixic acid & 442 & 408 (92.3) & 124 & 114 (91.9) \\
\hline MDR tested ${ }^{a}$ & 334 & $149(44.6)$ & 90 & $11(12.2)$ \\
\hline XDR tested ${ }^{b}$ & 283 & $2(0.7)$ & 75 & $0(0)$ \\
\hline
\end{tabular}

\section{TABLE 2: Antibiotic resistance patterns of Salmonella typhi and Salmonella paratyphi isolates}

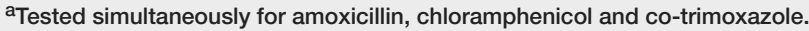

${ }^{\text {b}}$ Tested simultaneously for amoxicillin, chloramphenicol, co-trimoxazole, ciprofloxacin and ceftriaxone.

MDR, multidrug-resistant; XDR, extensively drug-resistant.

Most of the MDR Salmonella strains were sensitive to third-generation cephalosporins and imipenem. XDR S. typhi strains were all imipenem sensitive (Table 3). 


\section{Cureus}

\begin{tabular}{|c|c|c|c|c|}
\hline & \multicolumn{2}{|c|}{ Salmonella typhi } & \multicolumn{2}{|c|}{ Salmonella paratyphi } \\
\hline & Cases tested & Sensitive & Cases tested & Sensitive \\
\hline & $\mathrm{n}$ & $n(\%)$ & $\mathrm{n}$ & n (\%) \\
\hline & \multicolumn{4}{|c|}{ Multidrug-resistant } \\
\hline Azithromycin & 100 & $29(29)$ & 6 & $1(16.7)$ \\
\hline Cefixime & 55 & $51(92.7)$ & 6 & $6(100)$ \\
\hline Ceftriaxone & 142 & $138(97.2)$ & 10 & $10(100)$ \\
\hline Ciprofloxacin & 142 & $10(7)$ & 11 & $0(0)$ \\
\hline \multirow[t]{2}{*}{ Imipenem } & 97 & $91(93.8)$ & 10 & $9(90)$ \\
\hline & \multicolumn{4}{|c|}{ Extensively drug-resistant } \\
\hline Azithromycin & 1 & $1(100)$ & - & - \\
\hline Imipenem & 2 & $2(100)$ & - & - \\
\hline
\end{tabular}

\section{TABLE 3: Antibiotic susceptibility pattern of MDR and XDR strains of Salmonella isolates}

There has been an overall decrease in first-line antibiotic resistance rates and an increase in thirdgeneration cephalosporins resistance rates from 2012 to 2018 in S. typhi isolates. There was a sharp rise in resistance rates of most antibiotics during 2016 and 2017 (Figure 2).

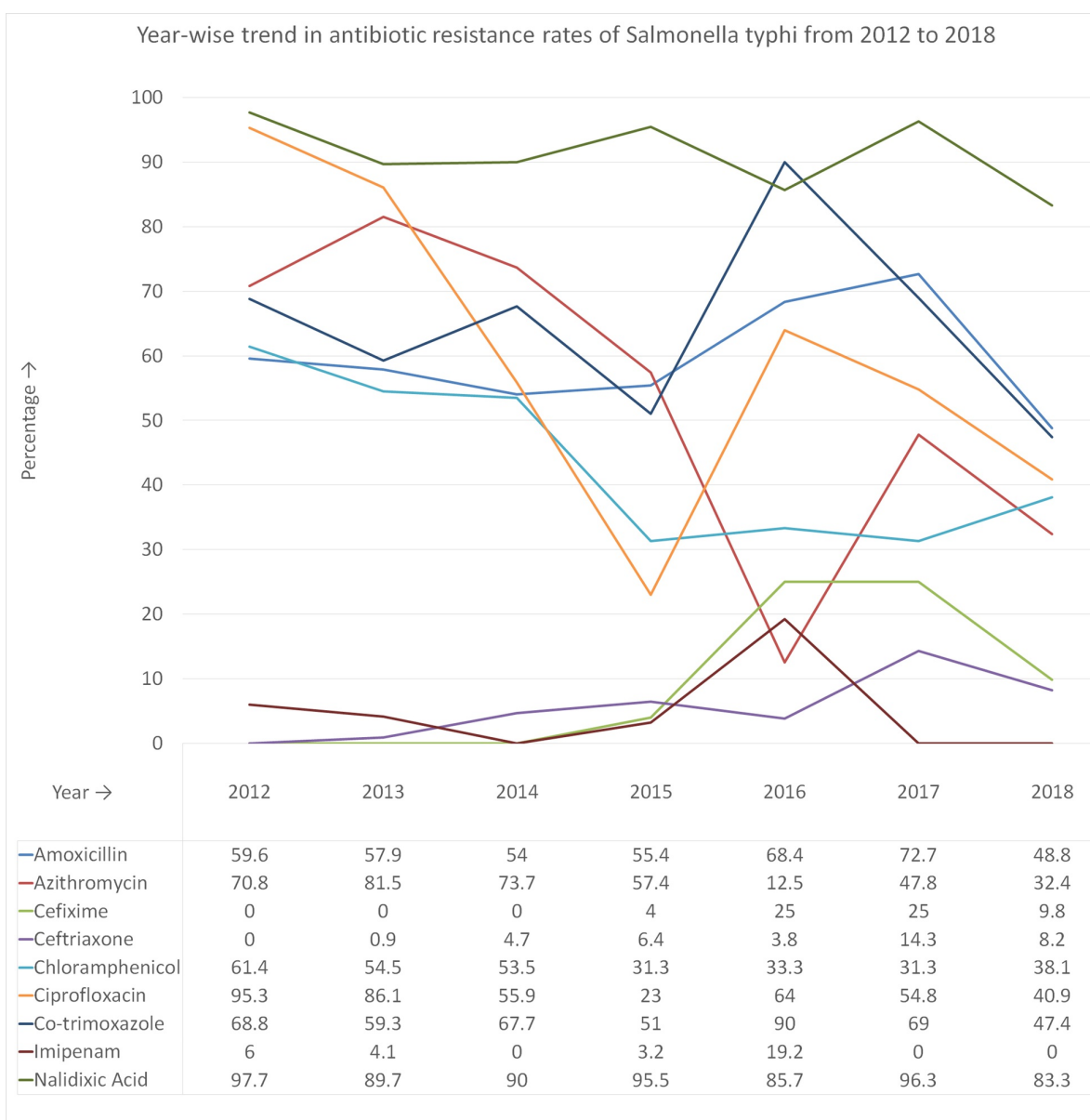

FIGURE 2: Year-wise trend in antibiotic resistance rates of Salmonella 


\section{Discussion}

Enteric fever is a foodborne and waterborne infectious disease caused by S. typhi (typhoid fever) and S. paratyphi (paratyphoid fever) [9]. Untreated infections can result in serious morbidity and even mortality. Most of the cases of enteric fever occur in Asia [1]. Enteric fever is endemic in the Indian sub-continent [10]. It is a major health problem in Pakistan. Antibiotic resistance is a major problem in the management of enteric fever. MDR and XDR cases of enteric fever have been reported $[5,11,12]$. The largest outbreak of ceftriaxone-resistant S. typhi was reported in Pakistan during 2017-2018 [12]. The knowledge of the local Salmonella antibiogram and antibiotic stewardship is crucial for the management of enteric fever. This study aimed to determine the antibiogram profile and compared year-wise trends in antibiotic resistance patterns of the cases of S. typhi and S. paratyphi in Pakistan Institute of Medical Sciences (PIMS) from 2012 to 2018.

In the present study, S. typhi was the predominant isolate from the patients with enteric fever in agreement with the previous studies $[2,13,14,15]$. Most of the cases were males. Other studies also reported a similar gender distribution $[1,13,16,17]$. Plausible explanation for this male predominance is the fact that males have more outdoor exposure and are more likely to consume street foods as compared to females in Pakistani society $[1,13,17]$. Street food consumption is an important risk factor for enteric fever [18]. A study conducted in Karachi reported a high prevalence of Salmonella carriage among food handlers working in food streets [19]. Analysis of age distribution reveals the clustering of cases in young adults (18-35 years). Sharvani et al. reported similar age distribution of the cases [1]. Young adults are more likely to be exposed to the pathogen because of the similar reasons that explain male prevalence. Clustering of cases was also observed during months of summer and monsoon in the present study. Khan et al. also reported a higher incidence during the summer season and attributed this trend to increased consumption of commercially prepared ice, chilled drinks and floods [10]. Sur et al. and Mohanty et al. reported a similar seasonal trend in India $[16,20]$.

Majority of the S. typhi isolates were resistant to all first-line antibiotics (amoxicillin, co-trimoxazole and chloramphenicol). Most of the S. typhi isolates including MDR strains were sensitive to cephalosporins and imipenem but resistant to ciprofloxacin and azithromycin. Qamar et al. and Khan et al. also reported a high prevalence of MDR Salmonella strains and a similar antibiotic resistance profile in Karachi and Hyderabad $[2,13]$. In contrast to our findings, Laghari et al. reported lower ceftriaxone and cefixime sensitivity rates, higher first-line antibiotic sensitivity rates and very low proportion of MDR strains among pediatric cases of S. typhi in Jamshoro [21]. Studies conducted in India and Nepal reported lower first-line antibiotic resistance rates and lower proportion of MDR strains among S. typhi $[1,22]$.

In this study, two XDR cases of S. typhi were identified; both were sensitive to imipenem, a carbapenem antibiotic. One of these cases was tested for and found to be sensitive to azithromycin as well. A study conducted on XDR S. typhi in Northern Pakistan reported antibiotic sensitivity only to azithromycin and meropenem [11]. Yousafzai et al. investigated the outbreak of ceftriaxone-resistant S. typhi in Hyderabad during 2016-2017 and reported a similar antibiotic sensitivity profile, that is, azithromycin, imipenem and meropenem [12].

In general, first-line antibiotic resistance rates were much lower among cases of S. paratyphi as compared to S. typhi thus accounting for lower proportion of MDR S. paratyphi in this study. This trend is consistent with the findings of Qamar et al. and Laghari et al. [13,21]. Furthermore, most of the S. paratyphi isolates were resistant to azithromycin, nalidixic acid, and ciprofloxacin but sensitive to cephalosporins and imipenem in our study. Sharvani et al. reported a similar trend in cephalosporin, ciprofloxacin and nalidixic acid resistance among cases of S. paratyphi in India [1]. No XDR strain of S. paratyphi was identified in our study.

Analysis of year-wise trends in antibiotic resistance profile of S. typhi from 2012 to 2018 reveals a sharp rise in resistance rates of most antibiotics during 2016 and 2017. Interestingly, this period coincides with the outbreak of ceftriaxone resistant S. typhi in Hyderabad during 2016-2017 [12,13]. However, there has been an overall decrease in resistance rates of first line antibiotics from 2012 to 2018. Qamar et al. reported a similar trend of reduction in first-line antibiotic resistance among cases of S. typhi from 2012 to 2014 in Hyderabad and Karachi [13]. There are reports of re-emergence of first-line antibiotic sensitivity in India and Nepal as well $[5,8]$. Explanation for this phenomenon is long-term reduced use of first-line antibiotics resulting in re-emergence of their sensitivity [13]. Moreover, there has been a fall in ciprofloxacin resistance rate and a slight rise in cephalosporin resistance rate among S. typhi isolates from 2012 to 2018 in our study; a trend also reported by Qamar et al. [13]. Over the last decade, ciprofloxacin use for enteric fever has been decreasing whereas cephalosporins use has been proportionately increasing thus explaining this trend.

The findings of this study recommend the use of third-generation cephalosporins as empirical agents of choice and for treatment of MDR cases of enteric fever; cefixime for oral treatment and ceftriaxone for parenteral therapy. Imipenem use should be reserved for the treatment of XDR cases of enteric fever. 
Azithromycin and ciprofloxacin should not be routinely used for empiric treatment owing to high drug resistance rates. Decreasing trend in first-line antibiotic resistance is promising; it may lead to reemergence of their sensitivity in the coming years. Therefore, antibiogram surveillance should be routinely performed to monitor antibiotic resistance patterns and to guide empirical treatment. Antimicrobial stewardship programs should be implemented to combat the growing problem of antibiotic resistance. General public should be educated about the risk factors and routes of transmission for enteric fever and encouraged to adopt measures of primary prevention. Health authorities should consider Salmonella vaccination campaigns to lessen the disease burden.

\section{Conclusions}

S. typhi accounted for most of the cases of enteric fever in the present study. Male predominance and seasonal variation with highest incidence during months of summer and monsoon were observed. Most of the S. typhi isolates were resistant to traditional first-line antibiotics and ciprofloxacin. Antibiotic resistance rates were lower in S. paratyphi isolates. This study recommends third-generation cephalosporins for empirical treatment and for treatment of MDR cases of enteric fever. Imipenem should be reserved for treatment of XDR Salmonella cases. Declining first-line antibiotic resistance rates is promising and it may lead to re-emergence of first-line antibiotic sensitivity at some point in future. Therefore, routine surveillance of Salmonella antibiogram and antibiotic stewardship is the need of the hour. Public health education campaigns and salmonella vaccination programs should be considered for primary prevention of the disease.

\section{Additional Information \\ Disclosures}

Human subjects: Consent was obtained by all participants in this study. Shaheed Zulfiqar Ali Bhutto Medical University issued approval No. F. 1-1/2015/ERB/SZABMU/459. Thank you for submitting your research proposal to the Ethical Review Board (ERB). After evaluation of your project, an unconditional permission is given to proceed with this project. . Animal subjects: All authors have confirmed that this study did not involve animal subjects or tissue. Conflicts of interest: In compliance with the ICMJE uniform disclosure form, all authors declare the following: Payment/services info: All authors have declared that no financial support was received from any organization for the submitted work. Financial relationships: All authors have declared that they have no financial relationships at present or within the previous three years with any organizations that might have an interest in the submitted work. Other relationships: All authors have declared that there are no other relationships or activities that could appear to have influenced the submitted work.

\section{Acknowledgements}

I am grateful to Almighty Allah, the most beneficent \& merciful for his countless blessings. I offer my special thanks to my supervisor Prof. Dr. Shajee Ahmed Siddiqui and to my parents and my family who have been very supportive.

\section{References}

1. Sharvani R, Hemavathi, Dayanand DK, Shenoy P, Sarmah P: Antibiogram of salmonella isolates: time to consider antibiotic salvage. J Clin Diagn Res. 2016, 10:DC06-DC08. 10.7860/JCDR/2016/18102.7753

2. Khan MI, Soofi SB, Ochiai RL, et al.: Epidemiology, clinical presentation, and patterns of drug resistance of Salmonella Typhi in Karachi, Pakistan. J Infect Dev Countries. 2012, 6:704-714. 10.3855/jidc.1967

3. Chang MS, Woo JH, Kim S: Management of typhoid fever - clinical and historical perspectives in Korea . Infect Chemother. 2019, 51:330-335. 10.3947/ic.2019.51.3.330

4. Britto CD, Wong VK, Dougan G, Pollard AJ: A systematic review of antimicrobial resistance in Salmonella enterica serovar Typhi, the etiological agent of typhoid. PLoS Negl Trop Dis. 2018, 12:e0006779. 10.1371/journal.pntd.0006779

5. Shrestha KL, Pant ND, Bhandari R, Khatri S, Shrestha B, Lekhak B: Re-emergence of the susceptibility of the Salmonella spp. isolated from blood samples to conventional first line antibiotics. Antimicrob Resist Infect Control. 2016, 5:22. 10.1186/s13756-016-0121-8

6. Azmatullah A, Qamar FN, Thaver D, Zaidi AK, Bhutta ZA: Systematic review of the global epidemiology, clinical and laboratory profile of enteric fever. J Glob Health. 2015, 5:020407. 10.7189/jogh.05.020407

7. Levine MM, Simon R: The gathering storm: is untreatable typhoid fever on the way? . mBio. 2018, 9:e0048218. 10.1128/mBio.00482-18

8. Bhatia JK, Mathur AD, Arora MM: Reemergence of chloramphenicol sensitivity in enteric fever . Med J Armed Forces India. 2007, 63:212-214. 10.1016/S0377-1237(07)80136-5

9. Kim S: Salmonella serovars from foodborne and waterborne diseases in Korea, 1998-2007: total isolates decreasing versus rare serovars emerging. J Korean Med Sci. 2010, 25:1693-1699. 10.3346/jkms.2010.25.12.1693

10. Naeem Khan M, Shafee M, Hussain K, et al.: Typhoid fever in paediatric patients in Quetta, Balochistan, Pakistan. Pak J Med Sci. 2013, 29:929-932. 10.12669/pjms.294.3251

11. Saeed N, Usman M, Khan E A: An overview of extensively drug-resistant Salmonella Typhi from a tertiary care hospital in Pakistan. Cureus. 2019, 11:e5663. 10.7759/cureus.5663

12. Yousafzai MT, Qamar FN, Shakoor S, et al.: Ceftriaxone-resistant salmonella typhi outbreak in Hyderabad 


\section{Cureus}

City of Sindh, Pakistan: high time for the introduction of typhoid conjugate vaccine. Clin Infect Dis. 2019, 68:S16-S21. 10.1093/cid/ciy877

13. Qamar FN, Yousafzai MT, Sultana S, et al.: A retrospective study of laboratory-based enteric fever surveillance, Pakistan, 2012-2014. J Infect Dis. 2018, 218:S201-S205. 10.1093/infdis/jiy205

14. Singh L, Cariappa MP: Blood culture isolates and antibiogram of Salmonella: experience of a tertiary care hospital. Med J Armed Forces India. 2016, 72:281-284. 10.1016/j.mjafi.2015.07.007

15. Das JK, Hasan R, Zafar A, et al.: Trends, associations, and antimicrobial resistance of salmonella typhi and paratyphi in Pakistan. Am J Trop Med Hyg. 2018, 99:48-54. 10.4269/ajtmh.18-0145

16. Mohanty S, Renuka K, Sood S, DAS BK, Kapil A: Antibiogram pattern and seasonality of Salmonella serotypes in a North Indian tertiary care hospital. Epidemiol Infect. 2006, 134:961-966. 10.1017/S0950268805005844

17. Khan M: A plausible explanation for male dominance in typhoid ileal perforation . Clin Exp Gastroenterol. 2012, 5:213-217. 10.2147/CEG.S36569

18. Gasem MH, Dolmans WM, Keuter MM, Diokomoeljanto RR: Poor food hygiene and housing as risk factors for typhoid fever in Semarang, Indonesia. Trop Med Int Health. 2001, 6:484-490. 10.1046/j.13653156.2001.00734.x

19. Siddiqui TR, Bibi S, Mustufa MA, Ayaz SM, Khan A: High prevalence of typhoidal Salmonella enterica serovars excreting food handlers in Karachi-Pakistan: a probable factor for regional typhoid endemicity. J Health Popul Nutr. 2015, 33:27. 10.1186/s41043-015-0037-6

20. Sur D, Barkume C, Mukhopadhyay B, Date K, Ganguly NK, Garrett D: A retrospective review of hospitalbased data on enteric fever in India, 2014-2015. J Infect Dis. 2018, 218:S206-S213. 10.1093/infdis/jiy502

21. Laghari GS, Hussain Z, Hussain SZM, Kumar H, Uddin SMM, Haq A: Antimicrobial susceptibility patterns of salmonella species in Southern Pakistan. Cureus. 2019, 11:e4379. 10.7759/cureus.4379

22. Khanal B, Sharma SK, Bhattacharya SK, Bhattarai NR, Deb M, Kanungo R: Antimicrobial susceptibility patterns of Salmonella enterica serotype typhi in eastern Nepal. J Health Popul Nutr. 2007, 25:82-87. 